\title{
Life history traits of the temperate mini-maximalist Idiosepius notoides, (Cephalopoda: Sepioidea)
}

\author{
Sean R. Tracey* ${ }^{\ddagger}$, Mike A. Steer ${ }^{\dagger}$ and Gretta T. Pecl* \\ *Marine Research Laboratories, Tasmanian Aquaculture and Fisheries Institute, University of Tasmania, Private Bag 49, Hobart, \\ Tasmania, 7001, Australia. 'School of Aquaculture, Tasmanian Aquaculture and Fisheries Institute, University of Tasmania, Private Bag \\ 370, Launceston, Tasmania, 7250, Australia. ${ }^{\ddagger}$ Corresponding author, e-mail: stracey@utas.edu.au
}

\begin{abstract}
Age, growth and maturation of the temperate 'mini-maximalist' Idiosepius notoides from Tasmania is described and compared with those of its tropical congener Idiosepius pygmaeus. Using statolith increment analysis, growth of $I$. notoides was best described by a power curve with a maximum age of 115 days recorded. Males have a shorter lifespan than females, however growth rates were similar between the sexes. Idiosepius notoides grows to a larger size than its tropical counterpart. Onset of maturity in I. notoides occurred at an age of approximately 68 days for males and 88 days for females compared to 45-60 days for I. pygmaeus. Size at onset of sexual maturity was analogous between the two species, with males mature at approximately $6.5 \mathrm{~mm}$ mantle length (ML) and females at $14.0 \mathrm{~mm}$ ML. Idiosepius notoides, like I. pygmaeus, is a small short-lived sepioid with significant gender dimorphism and the capacity to spawn multiple times throughout its short life. This research supports the concept of similar cephalopod species living longer and growing larger in cooler environments.
\end{abstract}

\section{INTRODUCTION}

Cephalopod life history traits are highly variable, particularly, growth rates and age/size at maturation. This variability is largely attributed to the indeterminate growth patterns typical of cephalopods where animals exhibit plastic, continuous growth and are capable of responding to a variety of biotic and abiotic factors. The diverse array of growth functions used to describe cephalopod growth in wild populations suggests many divergent patterns of growth, both inter- and intra-specifically. There is significant evidence to suggest that temperature has a dramatic effect on the growth of squid (Forsythe, 1993) with species that exhibit a broad latitudinal distribution expected to demonstrate different life cycles from north to south (Hanlon et al., 1983). In tropical waters there is relatively little seasonal variation and sub-annual life cycles generally occur (Jackson, 1990; Jackson \& Choat, 1992). However, in temperate waters squid grow larger, older, reproduce later, and are individually more fecund (O’Dor \& Coelho, 1993).

The genus Idiosepius comprises seven species of which two inhabit the coastal waters of Australia. Idiosepius notoides is found in the temperate waters of Tasmania and the south-east coast of Australia (Norman, 2000). Relatively little is known of the life history strategy of this temperate sepioid in comparison to its much-studied tropical relative, Idiosepius pygmaeus. Idiosepius pygmaeus extends from south-east Asia to northern Australia (Norman, 2000) and is a short-lived multiple spawner that can produce egg clutches totalling up to nine times its body weight (Lewis \& Choat, 1993) whilst still growing at a substantial rate (Jackson, 1989), hence being referred to as a 'mini-maximalist'. This study describes the body size, growth, lifespan, and maturation of the temperate Idiosepius notoides, using statolith derived age estimations, and explores the differences in life history between this temperate species and its tropical congener.

\section{MATERIALS AND METHODS}

A total of 110 Idiosepius notoides were collected from two sites on the east coast of Tasmania, Australia. Forty-two individuals were taken from Fortescue Bay $\left(43^{\circ} 08^{\prime} \mathrm{S}\right.$ $147^{\circ} 57 \mathrm{E}$ ) on 20 May 2000 and 68 from Coles Bay $\left(42^{\circ} 08^{\prime} \mathrm{S} 148^{\circ} 17^{\prime} \mathrm{E}\right)$ on the 14 June 2000 . Individuals were collected from drifting clumps of detached macro algae, found floating above shallow inshore sea-grass beds less than $15 \mathrm{~m}$ deep. The entire clump of macro algae was collected and $I$. notoides were shaken off into a dip net.

Individuals were placed on ice immediately after capture and processed within 5 hours. Dorsal mantle length (ML) was measured to the nearest millimetre with an optical graticule. Total body weight was weighed to the nearest milligram. Statoliths were removed from a sample of 40 individuals, following dissection to expose the cephalic chamber, where the calcareous statoliths were clearly visible within the statocyst. The statoliths were teased from the statocysts using a fine tipped probe. Once removed the statoliths were rinsed in $100 \%$ ethanol, airdried and embedded in thermoplastic resin (Crystal Bond $\left.{ }^{\circledR}\right)$. Each statolith was lightly ground on the anterior and posterior surfaces with wet $1200 \mu \mathrm{m}$ carborundum paper and polished with $0.05 \mu \mathrm{m}$ alumina powder on wet suede.

\section{Ageing}

Although daily increment periodicity has not been validated for Idiosepius notoides it has been confirmed for 
Table 1. Power functions describing length-weight relationships of individuals between sites and sexes, and between individuals' weight and length at age for males and females.

\begin{tabular}{|c|c|c|c|c|c|c|}
\hline Test & $\mathrm{a}$ & $\mathrm{a}(\mathrm{SE})$ & $\mathrm{b}$ & $\mathrm{b}(\mathrm{SE})$ & $r^{2}$ & $\mathrm{~N}$ \\
\hline Length-weight (males) & 0.582 & 0.46 & 2.45 & 0.21 & 0.89 & 54 \\
\hline Length-weight (females) & 0.883 & 0.21 & 2.28 & 0.09 & 0.93 & 49 \\
\hline Weight at age (males) & 0.0002 & 0.0007 & 3.07 & 1.27 & 0.32 & 17 \\
\hline Weight at age (females) & 0.0000007 & 0.0000004 & 4.76 & 0.74 & 0.73 & 15 \\
\hline Length at age (males) & 0.0028 & 0.007 & 1.85 & 0.68 & 0.36 & 17 \\
\hline Length at age (females) & 0.0005 & 0.0009 & 2.26 & 0.37 & 0.70 & 15 \\
\hline
\end{tabular}

I. pygmaeus (Jackson, 1989). It was assumed due to the similar anatomy of the two species and the similar growth pattern, that daily periodicity in statolith increments was valid for I. notoides. Statolith increments were counted using a compound light microscope at $400 \times$ magnification with a transmitted polarized light source. The image was digitally projected to the computer based image analysis software 'Image Prot' to give a more detailed view of the statolith structure. Increments were counted along the axis from the natal ring to the dorsal dome. Counts were made by two independent readers, with no reference to length or weight data to yield an index of reader precision. Age was calculated from the average value of all counts. If a count differed by more than $10 \%$ a third count was performed and the outlier discarded.

The average per cent error (APE) was calculated for both the within and between reader age estimates using the formulae of Beamish \& Fournier (1981):

$\mathrm{APE}=\frac{100}{\mathrm{~N}} \sum_{j=1}^{\mathcal{N}}\left[\frac{1}{R} \sum_{i=1}^{R} \frac{\left|X_{i j}-X_{j}\right|}{X_{j}}\right]$

where: $\mathrm{N}$ is the number of individuals aged, $R$ is the number of times individuals are aged, $X_{i j}$ is the $i$ th determination of the $j$ th individual, $X_{j}$ is the average estimated age of the $j$ th individual.

\section{Growth}

Exponential, linear, power, logistic and von Bertalanffy curves were fitted to the size-at-age data. The best fitting curve was determined by a combined examination of residuals for any systematic pattern and the degree of variability explained by the coefficient of determination $\left(r^{2}\right)$. Instantaneous growth rate was calculated for each individual averaged over its lifetime using the equation given in Forsythe \& Van Heukelem (1987):

$G=\left(\frac{\ln w_{2}-\ln w_{1}}{t_{2}-t_{1}}\right) \times 100$

where: $G$ is the per cent increase in body weight per day, $t_{1}$ is time at hatching, $t_{2}$ is time at capture, $w_{1}$ is wet weight $(\mathrm{mg})$ at $t_{1}$ and $w_{2}$ is wet weight $(\mathrm{mg})$ at $t_{2}$. Weight at time of hatching was estimated as $0.33 \mathrm{mg}$ based on the weight of five preserved I. pygmaeus hatchlings.

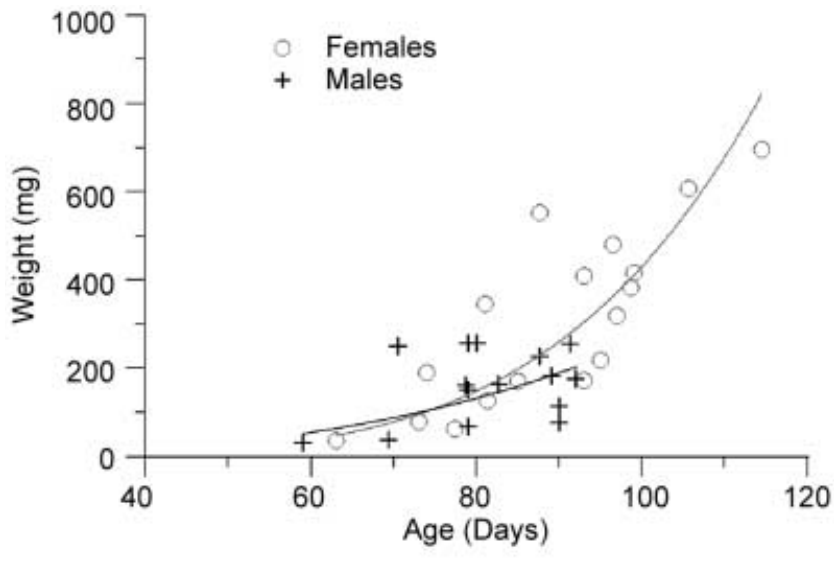

Figure 1. Weight at age relationships of male and female wild caught Idiosepius notoides from Tasmania.

\section{Histology}

The body of each individual was fixed in formaldehydeacetic acid-calcium chloride (FAACG) for one week before being stored in $70 \%$ ethanol. Specimens were processed to paraffin wax and sectioned whole longitudinally from the dorsal side mid-way through the animal (as in Lewis \& Choat, 1993). Serial sections were cut at $6 \mu \mathrm{m}$ through the midpoint of each individual on a rotary microtome at a consistent depth through the bodies. Sections were stained with Mayer's haematoxylin and Young's eosin, dehydrated and mounted on slides with dibutyl-phthalate-polystyrene-xylene (DPX). Animals were assigned a maturity stage according to the most advanced gamete stage present in the gonadal tissue in accordance with Sauer \& Lipinski (1990).

\section{Sea surface temperature}

Monthly average sea surface temperature (SST) of the area encompassing both sites at the time of sampling was calculated by analysing Reynolds SST data sourced from the NOAA-CIRES Climate Diagnostics Center, Boulder, Colorado, USA, via their Web site at http:// www.cdc.noaa.gov/.

\section{Statistical analysis}

Analysis of covariance (ANCOVA) was used to test for condition differences between the sexes based on the weight at length data. Average per cent growth rates between the sexes of aged squid were compared via a Student $t$-test. Association between growth of individuals 


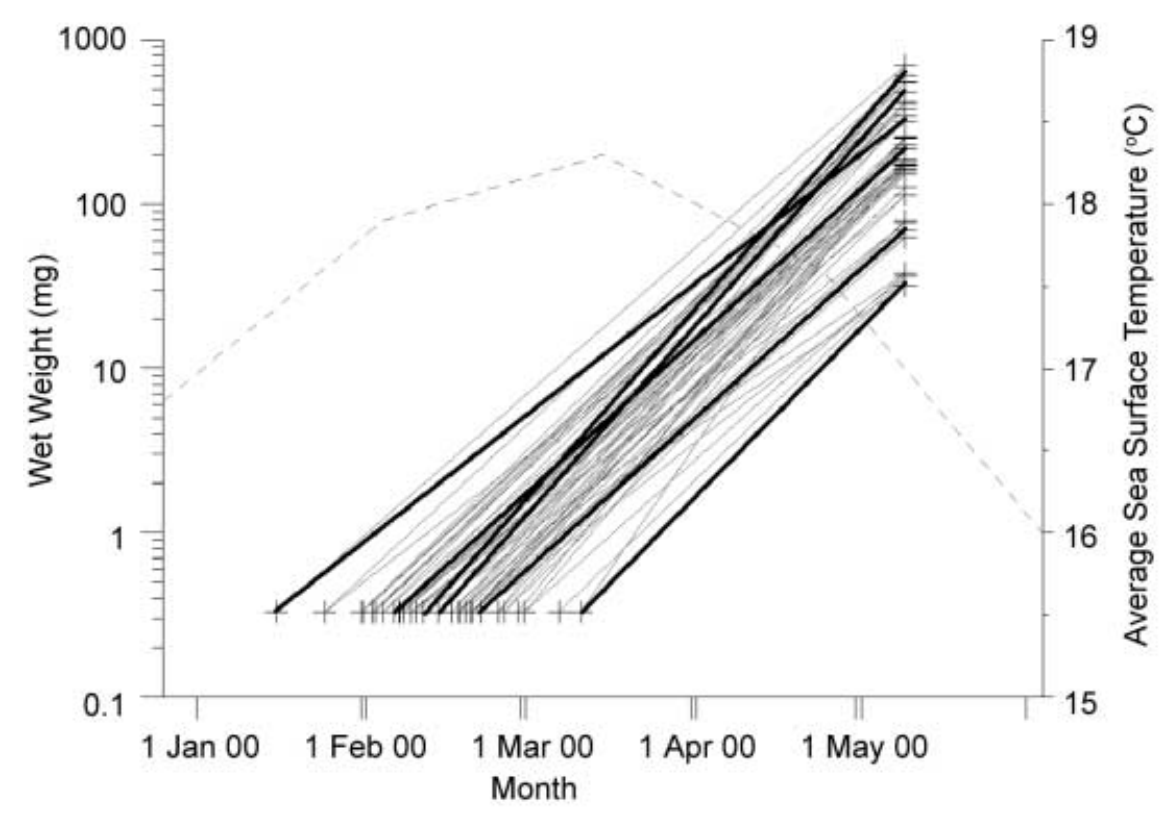

Figure 2. Individual growth trajectories of Idiosepius notoides (grey lines), solid black lines, selected randomly, illustrate the trends of growth at varying temperatures. Broken grey line represents average sea surface temperature.

Table 2. Summary of sex specific life history traits of Idiosepius notoides and Idiosepius pygmaeus.

\begin{tabular}{lcccr}
\hline & \multicolumn{2}{c}{ Idiosepius notoides } & & \multicolumn{2}{c}{ Idiosepius pygmaeus* $^{*}$ Female } \\
\cline { 2 - 5 } \cline { 4 - 5 } & Male & Female & Male & 18 \\
Maximum mantle length (mm) & 16 & 20 & 10 & 655 \\
Maximum total weight $(\mathrm{mg})$ & 298 & 398 & 159 & 79 \\
Maximum age (days) & 91 & 115 & 67 & 60 \\
Growth rate (\% day ${ }^{-1}$ ) & 7.5 & 8.4 & 42 & \multirow{2}{*}{ Age at maturity } \\
\hline
\end{tabular}

*, from Jackson (1989).

from the back-calculated hatch dates and average SST were tested using Pearson's linear correlation.

\section{RESULTS}

Idiosepius notoides ranged in length from 3.5 to $20.0 \mathrm{~mm}$ $\mathrm{ML}$ and 5.0 to $16.0 \mathrm{~mm}$ ML for females and males respectively. Females were significantly heavier than males (398 $\mathrm{mg} \pm 30 \mathrm{mg}$ SE, $\mathrm{N}=49$ and $298 \mathrm{mg} \pm 19 \mathrm{mg}$ SE, $\mathrm{N}=54$ respectively) when individuals from both sites were grouped $\left(t_{101}=2.95, P<0.01\right)$, suggesting a distinct size dimorphism between the sexes.

The length-weight relationships were best described by a power function (Table 1). No significant difference was detected in the slope $(F=1.35, P>0.05)$, or the intercept $(F=0.08, P>0.05)$ in the male and female length-weight comparisons, suggesting no difference in the rate of increase in weight with length.

\section{Ageing}

The index of APE was $2.49 \%$ for the main reader and $3.05 \%$ for the secondary reader. Readings by both the main and secondary reader were positively skewed toward higher counts on the second read compared to the initial count by the main reader. The maximum age estimates recorded were 115 days for a female $(\mathrm{ML}=19.8 \mathrm{~mm})$ and 91 days for a male $(\mathrm{ML}=10.6 \mathrm{~mm})$. A power relationship best described the increase in both length and weight (Figure 1) as a function of age for both the female and male populations (Table 1). The growth curves of each sex did not differ significantly by slope (ANCOVA; $F=1.29$, $P>0.05)$ nor intercept (ANCOVA; $F=0.90, P>0.05)$. The average per cent increase in body weight for females $\left(7.4 \% \mathrm{~d}^{-1}\right)$ was not significantly different from the males $\left(7.5 \% \mathrm{~d}^{-1}\right) \quad\left(t_{20}=-0.16, P>0.05\right)$. There was a significant linear correlation (Pearson's; $r=0.43, \mathrm{~N}=32, P<0.05$ ) between back-calculated hatch date and the growth trajectory of aged individuals (Figure 2). This relationship was further explored, revealing a significant positive correlation between the average sea surface temperature at the estimated time of hatching and growth rate (Pearson's; $r=0.34, \mathrm{~N}=32, P_{\text {(one-tailed) }}<0.05$ ).

\section{Maturation}

Males matured at a smaller size than females. Only two males (5.2 and $8.1 \mathrm{~mm} \mathrm{ML)} \mathrm{sampled} \mathrm{were} \mathrm{immature.} \mathrm{In}$ contrast, $50 \%$ maturity was reached at $14.5 \mathrm{~mm}$ and $100 \%$ at $18.0 \mathrm{~mm}$ for females. The youngest aged female 
that was found to have fully developed gametes was 88 days old. A summary of life history traits displayed by Idiosepius notoides and Idiosepius pygmaeus are described in Table 2.

\section{DISCUSSION}

Idiosepius notoides is a small, short-lived sepioid with a high degree of sexual dimorphism in both size and longevity. In this study males and females displayed similar growth rates with females achieving greater body length and weight by living approximately $25 \%$ longer than males. Mature female I. notoides commit a substantial energetic investment into reproduction, with the majority of the body cavity occupied by large nidamental glands and numerous large eggs. Consequently, it is not surprising that the two sexes appear to have contrasting life history strategies. Males attained maturity at half the size of females and at a younger age. Mature I. notoides females had eggs at all stages of development present in the ovary with the exception of stage five oocytes. Captive studies have shown that stage five oocytes are only retained for a matter of minutes in Idiosepius pygmaeus immediately prior to oviposition, and the ovaries of mature individuals contain eggs at all other stages of development. The link between continuous oocyte development and the capacity to spawn multiple clutches was confirmed through cultured rearing experiments (Lewis \& Choat, 1993). Reproductive output in both species is evident in the latter third of the lifespan, which has been defined as an attribute typical of a multiple spawner. As I. notoides has a very similar reproductive anatomical constitution to its tropical congener the likelihood of $I$. notoides being capable of multiple spawning is strong.

This study provides further evidence to support the theory of temperate cephalopod species growing larger, maturing later, and living longer than related species inhabiting warmer climes (Jackson \& Choat, 1992). Although the size at maturity of both sexes of the temperate $I$. notoides was analogous to that of its tropical congener I. pygmaeus, age at maturity differed between the two species. Male and female I. notoides achieved maturity approximately 20 days later compared with I. pygmaeus. Females of both species grow significantly larger than males; interestingly this is achieved by two distinct processes. Idiosepius pygmaeus females attain larger sizes than males via a comparatively faster growth rate (Jackson, 1989) whereas I. notoides females achieve this by increased longevity.

The link between the environment and growth in cephalopods is becoming more evident through the advances and the availability of oceanographic data. The importance of small changes in temperatures during the early life stage of cephalopod growth has been demonstrated to have a dramatic effect on an individual's growth trajectory and hence age and size at maturity (Forsythe, 1993). Growth in I. notoides also appears to be directly influenced by the temperature into which juveniles hatch. Individuals that hatched during warmer weeks grew faster on average than those hatched during cooler weeks.
Consequently, life history parameters such as growth rates, age and size at maturity, and final body size may show a high degree of spatial and temporal variability.

The results of this study highlight the complexities associated with Idiosepius notoides population dynamics and the complicated linkage between subsequent generations. As a result of a maximum lifespan of 115 days, and the variable nature of Tasmanian waters, an individual is likely to experience environmental conditions vastly different than previous generations. Further complicating the population dynamics, cross-generational spawning could result as a function of dimorphism between the longevity of males and females, and the extended spawning period of both sexes. The plasticity in the life history strategies of cephalopods is an integral mechanism in maintaining survivorship of populations by, allowing continuous response to varying biotic and abiotic variables.

We wish to thank Simon Willcox for assistance with sampling and an external referee for constructive comments.

\section{REFERENCES}

Beamish, R.J. \& Fournier, D.A., 1981. A method for comparing the precision of a set of age determinations. Canadian Fournal of Fisheries and Aquatic Sciences, 38, 982-983.

Forsythe, J.W., 1993. A working hypothesis of how seasonal temperature change may impact the field growth of young cephalopods. In Recent advances in cephalopod fisheries biology (ed. T. Okutani et al.), p. 143. Tokyo: Tokai University Press.

Forsythe, J.W. \& Van Heukelem, W.F., 1987. Growth. Cephalopod life cycles. Vol. II. Comparative reviews (ed. P. R. Boyle), pp. 135-156. London: Academic Press.

Hanlon, R.T., Hixon, R.F. \& Hulet, W.H., 1983. Survival, growth, and behaviour of the loliginid squids Loligo plei, Loligo pealei, and Lolliguncula brevis (Mollusca: Cephalopoda) in closed sea water systems. Biological Bulletin. Marine Biological Laboratory, Woods Hole, 165, 637-685.

Jackson, G.D., 1989. The use of statolith microstructures to analyze life-history events in the small tropical cephalopod Idiosepius pygmaeus. Fishery Bulletin, 87, 265-272.

Jackson, G.D., 1990. The use of tetracycline staining techniques to determine statolith growth ring periodicity in the tropical loliginid squids Loliolus noctiluca and Loligo chinensis. Veliger, 33, 389-393.

Jackson, G.D. \& Choat, J.H., 1992. Growth in tropical cephalopods: an analysis based on statolith microstructure. Canadian Journal of Fisheries and Aquatic Sciences, 49, 218-228.

Lewis, A.R. \& Choat, J.H. 1993. Spawning mode and reproductive output of the tropical cephalopod Idiosepius pygmaeus. Canadian Fournal of Fisheries and Aquatic Sciences, 50, 20-28.

Norman, M., 2000. Cephalopods, a world guide. Germany: Conch Books.

O’Dor, R.K. \& Coelho, M.L., 1993. Big squid, big currents and big fisheries. In Recent advances in cephalopod fisheries biology (ed. T. Okutani et al.), pp. 385-396. Tokyo: Tokai University Press.

Sauer, W.H.H. \& Lipinski, M.R., 1990. Histological validation of morphological stages of sexual maturity in Chokka squid Loligo vulgaris reynaudii D'orb (Cephalopoda: Loliginidae). South African Fournal of Marine Science, 9, 189-200.

Submitted 12 December 2002. Accepted 26 October 2003. 